\title{
Ultrashort pulse formation and evolution in mode-locked fiber lasers
}

\author{
M. Baumgartl • B. Ortaç • T. Schreiber · J. Limpert • \\ A. Tünnermann
}

Received: 18 April 2011 / Revised version: 9 May 2011 / Published online: 21 July 2011

(C) Springer-Verlag 2011

\begin{abstract}
Passive mode-locking in fiber lasers is investigated by numerical and experimental means. A nondistributed scalar model solving the nonlinear Schrödinger equation is implemented to study the starting behavior and intra-cavity dynamics numerically. Several operation regimes at positive net-cavity dispersion are experimentally accessed and studied in different environmentally stable, linear laser configurations. In particular, pulse formation and evolution in the chirped-pulse regime at highly positive cavity dispersion is discussed. Based on the experimental results a route to highly energetic pulse solutions is shown in numerical simulations.
\end{abstract}

\section{Introduction}

Lasers have been the subject of extensive research and development over the last 50 years, driven by their huge application potential. However, laser oscillators may be likewise

\footnotetext{
M. Baumgartl $(\bowtie) \cdot$ J. Limpert · A. Tünnermann Institute of Applied Physics, Friedrich-Schiller-Universität Jena, Albert-Einstein-Str. 15, 07745 Jena, Germany

e-mail: martin.baumgartl@uni-jena.de

Fax: +49-3641-947802
}

M. Baumgartl · J. Limpert · A. Tünnermann

Helmholtz-Institute Jena, Max-Wien-Platz 1, 07743 Jena,

Germany

\section{B. Ortaç}

UNAM-Institute of Materials Science and Nanotechnology,

Bilkent University, 06800 Bilkent, Ankara, Turkey

e-mail: ortac@unam.bilkent.edu.tr

T. Schreiber · A. Tünnermann

Fraunhofer Institute for Applied Optics and Precision

Engineering, Albert-Einstein-Str. 7, 07745 Jena, Germany regarded as a very prominent example of a nonlinear optical dissipative system where the nonlinear dynamics of the optical field is primarily governed by its energy exchange with the environment. Fiber lasers, in particular, exhibit complex ultrafast temporal dynamics, as nonlinear effects are enhanced due to the high intensities in the fiber core and the long interaction length. Hence there is furthermore strong research interest in fundamental aspects of these highly applicable systems. In the temporal domain fiber lasers are an easily accessible experimental laboratory for these investigations, because transverse field confinement separates the temporal from the spatial dynamics, the interplay of which evoke a complex spatio-temporal evolution in other laser types. The detailed understanding of the laser dynamics allows for improving the performance of these laser systems by increasing their flexibility to create complex shapes of ultrashort pulses.

In the following a study of different operation regimes of mode-locked fiber laser resonators is presented. In the first part a numerical model is presented, which is suitable to describe pulse formation and evolution in mode-locked fiber lasers. Within this part firstly, the influence of initial conditions to access different attractors of an ultrashort pulse fiber laser for fixed system parameters is investigated. Furthermore a variation of the system parameters is done within the scope of different regimes of mode-locking. As a result, the pulse shaping in the net-normal-dispersion regime is revised. In the second paragraph pulse generation in an environmentally stable all-fiber laser configuration is demonstrated and investigated in the stretched-pulse and wavebreaking free regimes. The last paragraph deals with passive mode-locking at high positive net-cavity dispersion in the chirped-pulse regime. This new regime is experimentally and numerically investigated. Based on the experimental results pulse energy scaling within this approach is studied in simulations. 


\section{Numerical modelling of mode-locked fiber lasers}

Steady state solutions of stable pulses in fiber oscillator systems exist due to the compensation of dispersion and nonlinearity but also dissipation and saturable gain. For theoretical modelling of these passively mode-locked lasers the Ginzburg-Landau equation (GLE) or several extensions are often used [1], where the actions within the cavity are averaged based in the assumption of small changes during one round trip [2]. These equations form stable solutions, which describe the outcome of the experimental laser for a large range of parameters quite well. Beside the research based on the GLE, the numerical approach of following the pulse inside a cavity by transmitting though each element has been developed and applied to Ti:Sa lasers [3]. Such onedimensional models with non-distributed parameters have been found to describe the experimental results of Ti:Sa lasers quite well with the drawback of lacking analytical access compared to the GLE approach. However, they could be used to study intra-cavity propagation dynamics, which are mainly determined by the net-cavity dispersion. For fiber lasers several regimes are known: Soliton mode-locking is characterized by negative net-cavity dispersion, where the pulse shape is that of a fundamental soliton $\left(\mathrm{sech}^{2}\right)$. This regime is well-known for Er-doped fiber lasers, where the fiber itself provides anomalous dispersion. Due to the soliton area theorem, the output energies of such lasers are limited to $\sim 0.1 \mathrm{~nJ}$. In analogy to long haul communication lines, dispersion-managed mode-locking has been discovered and described, where the fiber dispersion is compensated and the net cavity dispersion is close to zero [4]. However, the break-through in terms of output energy of more than $1 \mathrm{~nJ}$ was obtained with the access of wave-breaking free modelocking (also termed self-similar mode-locking) operating in the normal-dispersion regime [5]. In fact, the large amount of nonlinear dispersive material in fiber lasers makes it to an interesting realization of a dissipative nonlinear system with a variety of possible pulse shaping mechanisms not yet fully understood.

In the following, we study different aspects of fiber lasers based on a non-distributed model, which makes fewer assumptions on the pulse changes during one round trip compared to the GLE approach and maintains the possibility of studying the intra-cavity pulse evolution. Our model is based on simulating every part of the oscillator (Fig. 1) separately by solving the nonlinear Schrödinger equation with the splitstep algorithm (see [6] for details).

For investigation within this paragraph we simulate the cavity scheme, which is shown in Fig. 1. The gain fiber is followed by the saturable absorber (SA) with an infinitely fast response time, which is appropriate for any absorber based on the Kerr effect. The losses and output coupling are summarized by a reduction of pulse energy by a factor of

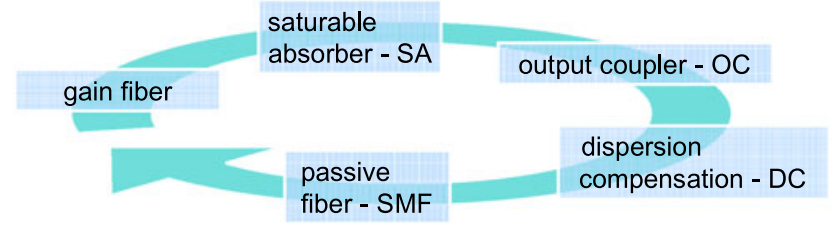

Fig. 1 Illustration of the fiber laser cavity elements used for the simulations

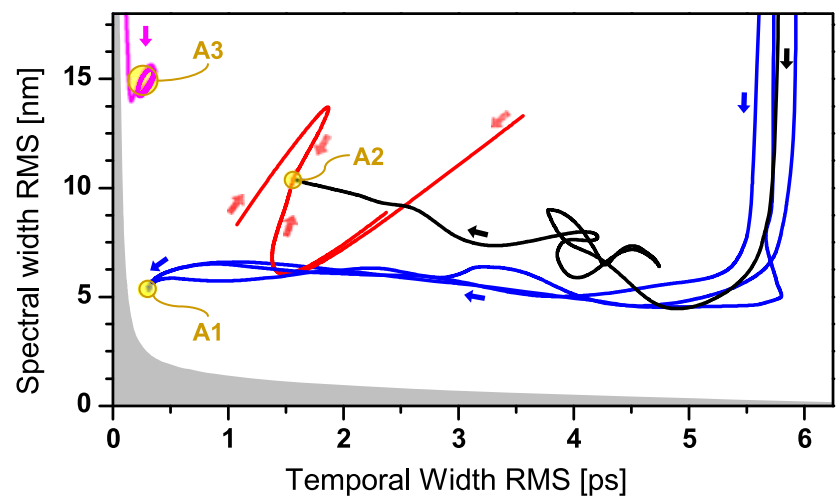

Fig. 2 Basin of attractors presented in the space of temporal and spectral width (RMS values) for the parameters of $E_{\text {sat }}=100 \mathrm{pJ}$ and $\beta_{2}=0.004 \mathrm{ps}^{2}$. The lines connect the measured values after each round trip. The arrows indicate the evolution from the initial condition to the attractor marked with yellow circles. (Gray: below transform-limited condition)

10 after the SA. The remaining power propagates through a dispersion compensation stage before entering the passive single mode fiber, which closes the loop. The detailed parameters for each element can be found in Table 1 in [6]. The net-cavity dispersion is only changed by the dispersion compensation all other elements remain with fixed dispersion.

\subsection{Initial conditions and basin of attraction}

To show the variety of attractors for a given set of laser parameters that can also be found for the non-distributed model, several simulations have been done. The net-cavity dispersion is fixed to $+0.004 \mathrm{ps}^{2}$ and the saturation energy to $E_{\text {sat }}=100 \mathrm{pJ}$. To access the different solutions, several initial conditions have been used and the transition to their attractor is shown in a Poincare map in Fig. 2. There, the RMS width of the spectral domain with respect to the temporal domain after each round trip (after the SMF) is plotted and gives access to the time-spectral relation of the pulse even if the complete phase cannot be shown by this graph. The blue and black graphs show the evolution starting from quantum noise, where each line starts from different noise. For the red lines, a Gaussian pulse with different initial chirp settings and spectral width is the initial condition. The purple line starts from a transform-limited Gaussian pulse with 
Fig. 3 Examples of the convergence to the different attractors in the temporal domain. (Linear scale: 0 1 max)
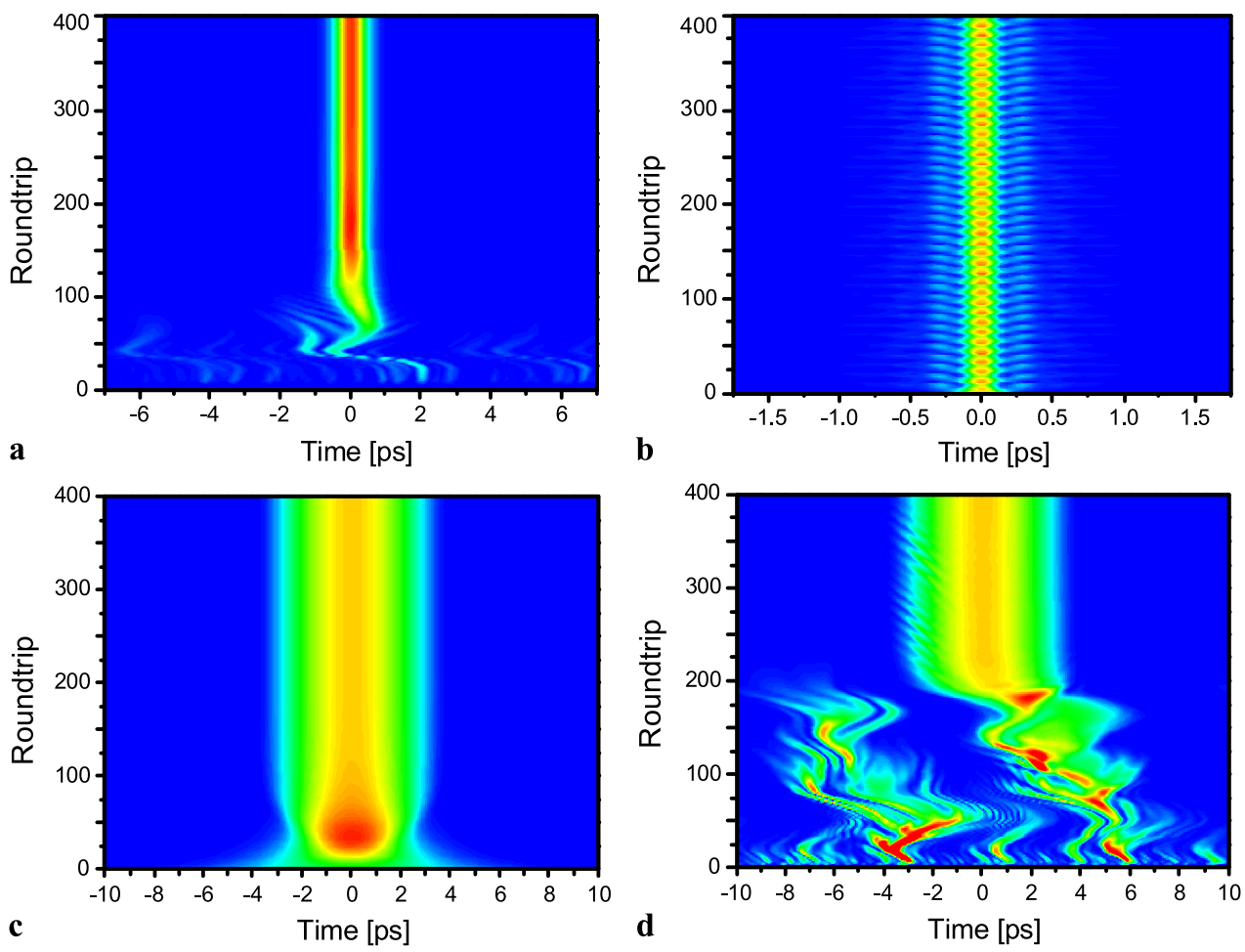

a spectral width of $27.13 \mathrm{~nm}$. All simulations have been checked to converge to the same attractor when repeated with higher temporal or spectral resolution. As a result of these simulations one can see that there are at least three different attractors accessible, which form the basin of attractors for the fixed set of laser parameters. The evolution of the initial conditions to these attractors is shown in Fig. 2 and Fig. 3. The first attractor (attractor A1) is obtained by the quantum noise initial conditions following the blue lines in Fig. 2 (see also Fig. 3(a)). Its parameters in front of the gain fiber are $\Delta \lambda_{\mathrm{RMS}}=5.45 \mathrm{~nm}$ and $\Delta T_{\mathrm{RMS}}=322 \mathrm{fs}$. With this solution, the pulse evolves in stretched-pulse mode intracavity. Interestingly, the black line also starts from quantum noise, however, the final solution is a linear chirped pulse with the parameters of $\Delta \lambda_{\mathrm{RMS}}=10.35 \mathrm{~nm}$ and $\Delta T_{\mathrm{RMS}}=$ $1.57 \mathrm{ps}$ in front of the gain fiber (Fig. 3(d)). This second attractor (attractor A2) is also obtained for the initial conditions of a stretched Gaussian pulse (Fig. 3(c)) shown as the red lines in Fig. 2 and is identified as a region of wavebreaking free intra-cavity pulse evolution. Finally, the third attractor (attractor A3) forms an oscillating solution (purple line in Fig. 2) shown in Fig. 3(b) in detail.

Another aspect of these numerical simulations is the stability of the attractors. All solutions discussed so far are stable, meaning that the stationary pulse does not change after several thousand round trips and even stabilizes if a small amount of noise is added to the solution.
2.2 Accessing different pulse regimes by variation of system parameters

In the previous section we showed that for a given set of parameters of the mode-locked laser, the numerical solution depends on the initial condition. In this section the different pulse regimes of the laser are analyzed by changing the parameters of $E_{\text {sat }}$ from $100 \mathrm{pJ}$ to $400 \mathrm{pJ}\left(100 \mathrm{pJ}\right.$ steps) and $\beta_{2}$ net from $-0.002 \mathrm{ps}^{2}$ to $+0.01 \mathrm{ps}^{2}\left(0.0005 \mathrm{ps}^{2}\right.$ steps $)$ for the initial condition of a transform-limited Gaussian pulse with $E_{0}=66 \mathrm{pJ}$ and $T_{0}=100 \mathrm{fs}$ in front of the SMF (all other parameters remain the same as above).

The temporal and spectral characterization of the obtained stable solutions is discussed in the following. In the subsection the focus lies on the regime of mode-locking and therefore the pulse shape especially for normal (positive) net-cavity dispersion. Figure 4(a) shows the temporal width (FWHM) after the SMF for the given set of parameters. Parameter settings where the solution did not converge are plotted in white. The corresponding spectral width (FWHM) is shown in Fig. 4(b).

For this range of parameters two fundamental different intra-cavity regimes are found and are directly visible in Fig. 4, where the regimes are distinguishable by the temporal width. For negative and small values of positive dispersion, the width after the SMF is quite small compared to the values obtained for larger positive net-cavity dispersion. Figure 5(a) and (b) shows the temporal intra-cavity pulse evolution for two representative points in the parameters 
space of the regimes. In Fig. 5(a), where $E_{\mathrm{sat}}=100 \mathrm{pJ}$ and $\beta_{2}=-0.002 \mathrm{ps}^{2}$, the pulse width has two minimum points, which is well-known for stretched-pulse mode-locking [4]. In contrast, for $E_{\mathrm{sat}}=400 \mathrm{pJ}, \beta_{2}=+0.0045 \mathrm{ps}^{2}$ Fig. 5(b) shows that only one minimum exists for the pulse width inside the cavity. The pulse spectrum and pulse shape is almost parabolic, thus, this regime is also known as self-similar mode-locking [5].

\subsubsection{Pulse shape for normal dispersion}

To investigate the pulse shaping in more detail, another parameter of the pulses temporal and spectral shape has been analyzed. The kurtosis $k$ has already been used to characterize parabolic pulses [7]. It is defined by (1), where $\mu_{4}$ is the fourth centered momentum and $\sigma^{4}$ the square of the variance. For parabolic shapes it has a value of $k=-0.86$ and $k=0$ for a Gaussian pulse shape.

$k=\frac{\mu_{4}}{\sigma^{4}}-3$

The temporal and spectral kurtosis is shown in Fig. 6(a) and $6(\mathrm{~b})$, respectively, for positive values of $\beta_{2 \text { net }}=0.0035 \cdots$ 0.01 and $E_{\text {sat }}=100 \cdots 400$ pJ, where Gaussian and quantum noise initial condition result converge to the same attractor. However, as can be seen in this graph, only for a small region around $\beta_{2 \text { net }} \sim 0.004$ and $E_{\text {sat }}=300 \cdots 400 \mathrm{pJ}$ the kurtosis is close to -0.86 in the temporal as well as the
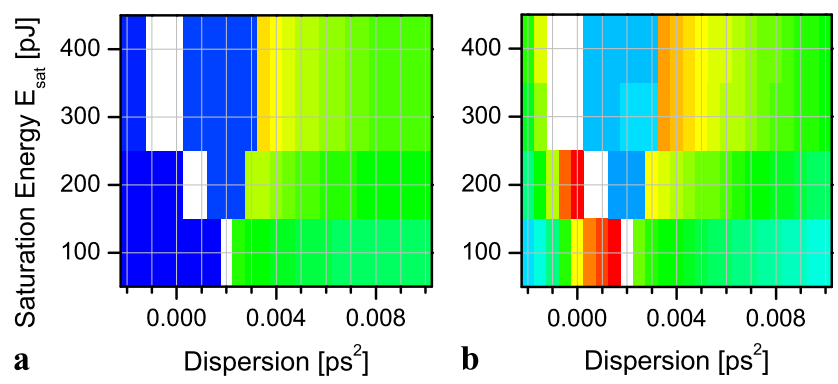

Fig. 4 (a) Pulse duration (FWHM) and (b) spectral bandwidth of the converged solution after the SMF. (Linear scale: $0 \square 10 \mathrm{ps}$ [white: unstable] $5 \mathrm{~nm} \quad 35 \mathrm{~nm}$ ) spectral domain. It indicates that only in this region the chirp is linear and images the parabolic shaped spectrum in the temporal domain. This is what is known from self-similar propagation [8]. It is also well-known that for a fast convergence to the self-similar evolution in the SMF inside the cavity, the pulse parameters of the pulse entering the SMF have to be optimum. For passive fibers such a condition was discussed by [9]. It is therefore clear that only a limited range of intra-cavity pulse conditions lead to pulses evolving truly self-similar in the SMF and explains the small region of equal kurtosis in the temporal and spectral domain.

Figure 7(a) again shows the deviation from a perfect parabolic shape in the temporal domain indicated by the kurtosis values in Fig. 6 for three representative parameters: $E_{\text {sat }}=400 \mathrm{pJ}, \beta_{2 \text { net }}=0.0045 \mathrm{ps}^{2}$ being in the region of a kurtosis close to -0.86 in the spectral and temporal domain; and $E_{\text {sat }}=400 \mathrm{pJ}, \beta_{2 \text { net }}=0.0045 \mathrm{ps}^{2}$ and $E_{\mathrm{sat}}=100 \mathrm{pJ}$ and $\beta_{2 \text { net }}=0.01 \mathrm{ps}^{2}$ far outside this region. To prove if a linear chirp is obtained within and outside the region of self-similar evolution Fig. 7(b-d) shows the spectrograms of the pulse obtained after the SMF for the same three sets of parameters. For Fig. 7(c) and (d) variations from the linear chirp in the pulse and spectral wings are obvious. It proves that only for the aforementioned self-similar regime, truly linear chirped pulses are obtained. Such pulses are especially of practical relevance due to the advantages for highpeak power amplification [10]. However, in the whole wavebreaking-free regime, the linear chirp is dominant in the solution and recompression of the pulses close to transformlimit should be possible.

In summary it is important to revise and keep in mind that the "wave-breaking-free mode-locking" is the more general term and embeds "self-similar mode-locking", where a linear chirped parabolic pulse is indeed obtained due to self-similar evolution in the SMF. Furthermore, if other cavity geometries (e.g. linear cavities [11]) are considered one has to check again if self-similar evolution is possible at all within a wave-breaking-free regime.
Fig. 5 Intra-cavity pulse evolution for (a) $E_{\mathrm{sat}}=100 \mathrm{pJ}$, $\beta_{2}=-0.002 \mathrm{ps}^{2}$ and

(b) $E_{\mathrm{sat}}=200 \mathrm{pJ}$, $\beta_{2}=+0.003 \mathrm{ps}^{2}$. (Logarithmic scale: $-30 \mathrm{~dB} \quad(0 \mathrm{~dB})$

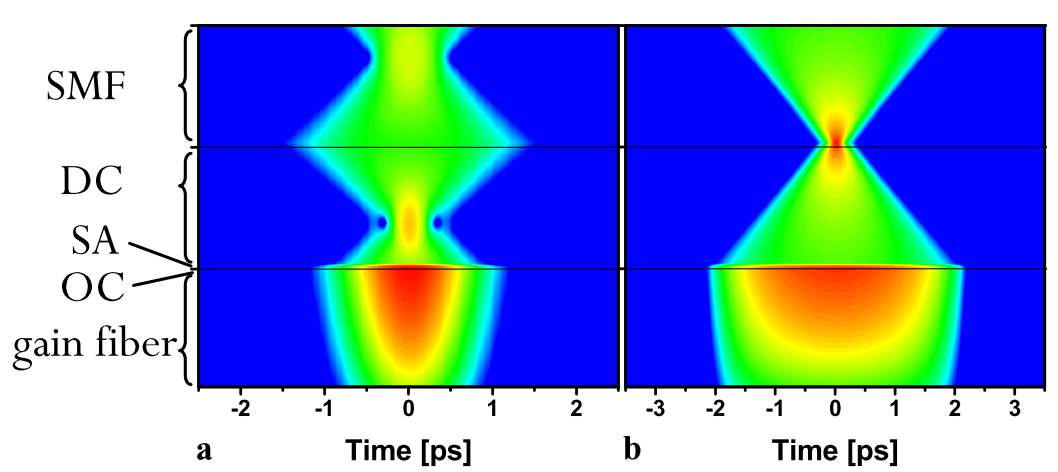




\section{Stretched-pulse and wave-braking-free fundamental mode-locked operation in an environmentally stable all-fiber laser configuration}

Whereas in the last paragraph general stability considerations and convergence to different attractors and access to different mode-locking regimes were discussed, this paragraph presents the implementation and investigation of the stretched-pulse and wave-braking-free regimes in a highly stable experimental laser setup, which is due to its reliability and robustness of high interest for ultrafast applications.

Lasers in stretched-pulse regime configuration possess a total-cavity dispersion close to zero. The pulse width experiences large variations per cavity round trip, with a change in the chirp sign. Stretched-pulse lasers with output energies from tens of picojoules to some nanojoules have been reported [12-14]. The most fundamental limitation to pulse energy in a fiber oscillator arises from wave-breaking phenomena because of large nonlinearities. More recently, wave-breaking is suppressed within the cavity by exploit-
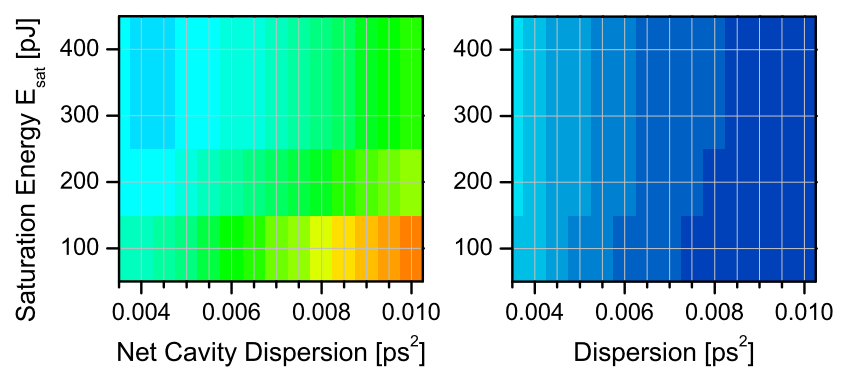

Fig. 6 Temporal and spectral kurtosis of the converged solution after the SMF. (Linear scale: $-1.1 \quad 0.2,-0.86$ ) ing self-similar pulse propagation, which is resistant to nonlinearity [15]. In the wave-breaking-free mode-locked fiber laser, the net-cavity dispersion is positive and the nonlinear pulse evolution in the normal group velocity dispersion (GVD) fiber segment is monotonic. The pulse accumulates a linear chirp, which is partially compensated at points in the cavity using a linear process (e.g. diffraction gratings). In the following, we discuss both experimental and numerical generation of wave-breaking-free and stretched pulses from an environmentally stable Yb-doped all-fiber laser. The generation and intra-cavity evolution of wave-breaking-free and stretched pulses are confirmed by a numerical analysis. Especially, we present the generation of pulses with a parabolic spectral shape in the stretched-pulse regime.

\subsection{Experimental setup}

The linear cavity (Fig. 8) is constructed with polarizationmaintaining single-mode fiber allowing for an environmentally stable configuration. Self-starting passive modelocking has been achieved through a semiconductor saturable-absorber mirror (SAM) which is directly glued to the fiber end-facet. A chirped fiber Bragg grating (CFBG) is employed for intra-cavity dispersion compensation. It provides a dispersion of $-0.19 \mathrm{ps}^{2}$ at $1035 \mathrm{~nm}$ and is also used as output coupler of the linear cavity. Further details are given in [16].

\subsection{Wave-breaking-free regime}

In the wave-breaking-free operation, the total fiber length inside the cavity is $4.9 \mathrm{~m}$ resulting in a second-order cavity
Fig. 7 (a) Temporal pulse profiles normalized in time to their FWHM in comparison with a perfect parabolic shape (black) shown in logarithmic scale. (Spectral profiles are not shown but exhibit similar features.) (b-d) Spectrograms of the steady state solutions. (Linear scale: 0 $\max )$
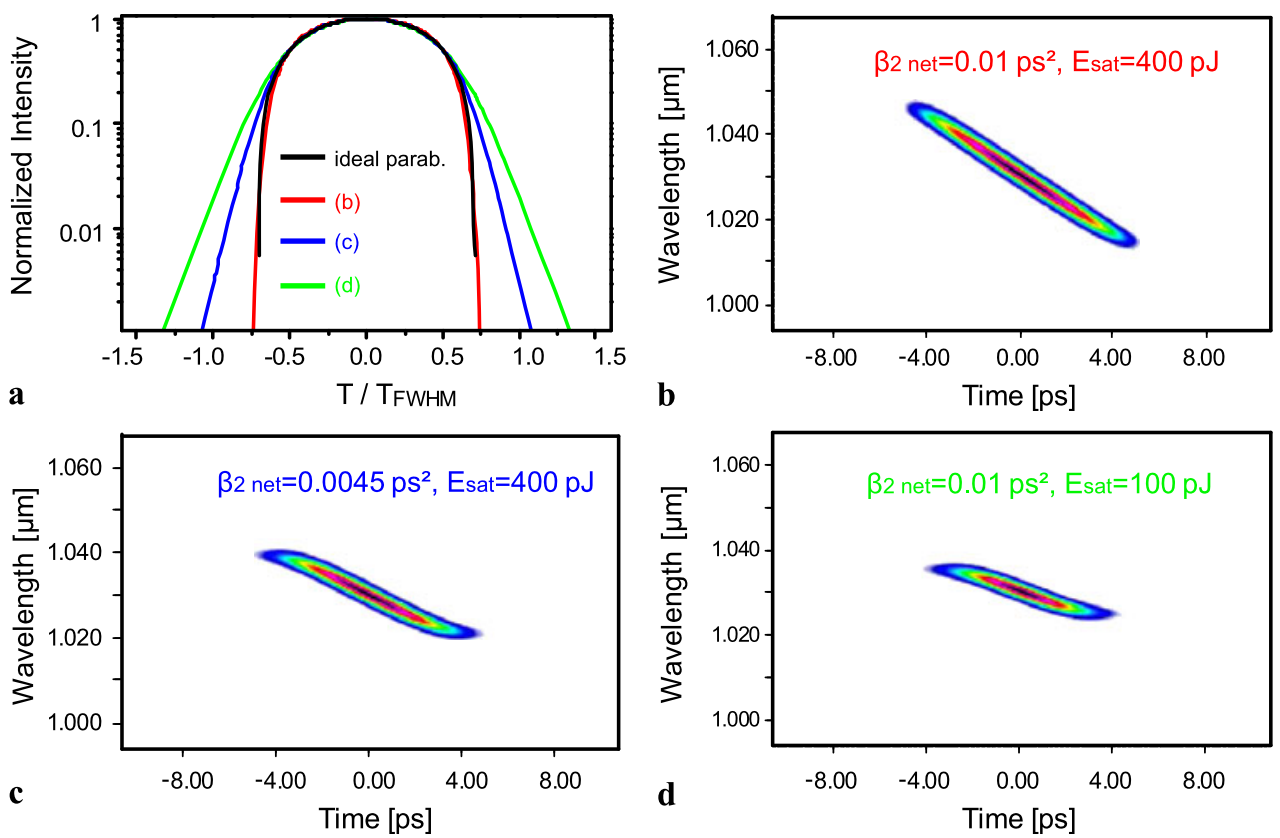


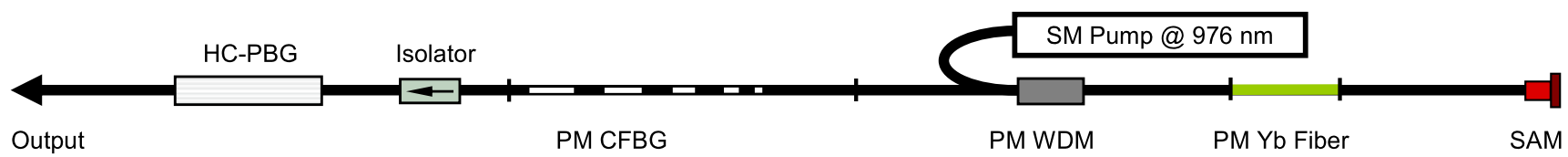

Fig. 8 Schematic representation of the polarization-maintaining $(P M)$ all-fiber laser with fiber-based extra-cavity pulse compression. SAM: saturable-absorber mirror, WDM: wavelength division multiplexer, $C F B G$ : chirped fiber Bragg grating, $H C$ - $P B G$ : hollow-core photonic-bandgap fiber

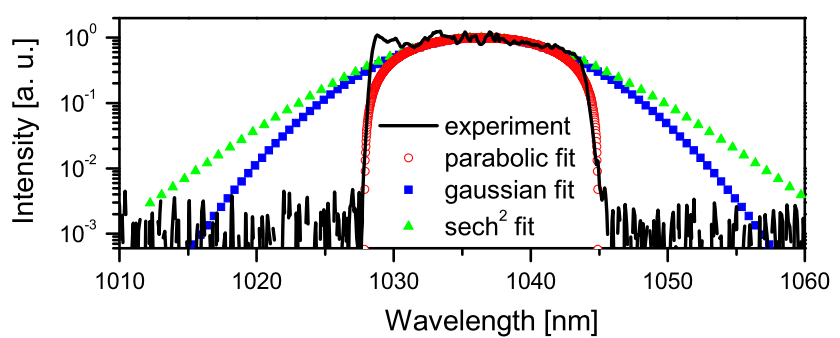

Fig. 9 Optical spectrum of the wave-breaking-free pulses in comparison with a parabolic, Gaussian and $\operatorname{sech}^{2}$ pulse shape
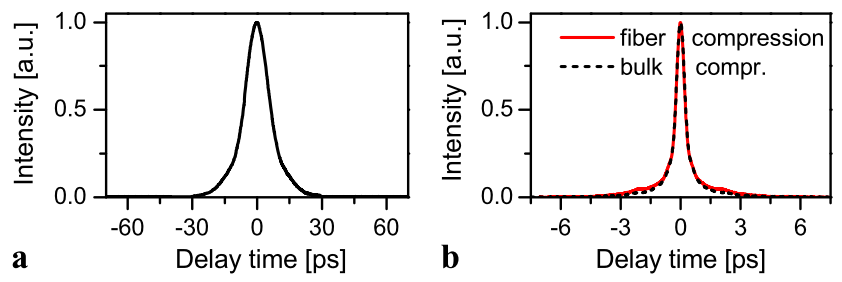

Fig. 10 (a) Autocorrelation trace of the chirped wave-breaking-free pulses. (b) Autocorrelation trace of the compressed pulses using a HC-PBG fiber compared to bulk grating compression

dispersion of $0.055 \mathrm{ps}^{2}$. The mode-locking regime is very stable and self-starting with the same characteristic of operation (spectrally and temporally) for equal pump power. Figure 9 shows the optical power spectrum together with different theoretical fits using sech ${ }^{2}$, Gaussian and parabolic profiles. The experimental spectrum presents the best agreement with the parabolic profile of $13.9 \mathrm{~nm}$ spectral width centered around $1035 \mathrm{~nm}$ mainly due to its steep edges. We attribute the residual modulation in the spectrum to interference effects by polarization mode mixing and intra-cavity splice issues. Depending on the pump power, we could observe such a spectral shape for a net-cavity dispersion of $0.048 \mathrm{ps}^{2}$ to $0.075 \mathrm{ps}^{2}$ by changing the passive fiber length.

Figure 10(a) shows the autocorrelation trace obtained directly at the laser output. The positively chirped output pulses possess an energy of $390 \mathrm{pJ}$ and have a duration of 15.4 ps (autocorrelation width of $16.4 \mathrm{ps}$ ). For comparison these pulses are compressed firstly by a grating pair outside the cavity. Subsequently, the compression is done by a hollow core photonic bandgap fiber (HC-PBG) allowing for a robust all-fiber configuration delivering ultrashort pulses. The fiber length corresponds to $-0.38 \mathrm{ps}^{2}$ of dispersion and has been optimized to obtain minimum pulse duration. The dispersion needed for pulse compression thus is much larger than the negative dispersion given by the CFBG. This proves that the pulses are always positively chirped inside the cavity with only one minimum per round trip located at the end of the anomalous GVD segment. An autocorrelation width as short as 328 fs (FWHM) has been obtained (Fig. 10(b)), which indicates a compression factor of more than 49 . The pulse duration can be calculated from the width of the autocorrelation by assuming a transform-limited wave-breakingfree spectrum of the compressed pulse and is evaluated to be $218 \mathrm{fs}$.

\subsubsection{Intracavity evolution}

The external compression already proved a positively chirped pulse propagating inside the cavity. To further confirm this statement and get insight into the pulse evolution, a numerical simulation has been performed with our nondistributed model. The parameters for each element are that of the experimental setup. The simulation started from quantum noise and after convergence, the pulses intra-cavity evolution is calculated. The result is shown in Fig. 11 where the propagation in the single-mode fiber and the gain fiber is scaled to an identical part of the cavity, whereas the saturable absorber and the dispersion compensation by the CFBG is done in a single step. One can clearly see that the pulse spectrum changes only slightly but shows steep edges as measured experimentally. The pulse duration increases until the dispersion compensation, which does not reverse the sign of the chirp meaning that only one minimum in pulse duration is found. The minimum pulse duration located at the end of the anomalous GVD segment is $1.8 \mathrm{ps}$. With these experimental and numerical results we can conclude that the laser works in a wave-breaking free regime, where the pulse has a positive linear chirp at each position in the cavity.

\subsection{Stretched-pulse regime}

As discussed in the previous section, we obtained a close to parabolic intensity spectral profile in the wave-breakingfree pulse regime for a large wide of normal net-cavity dispersion. It is now of particular interest to know if similar behavior can be obtained in the close to wave-breaking-free 

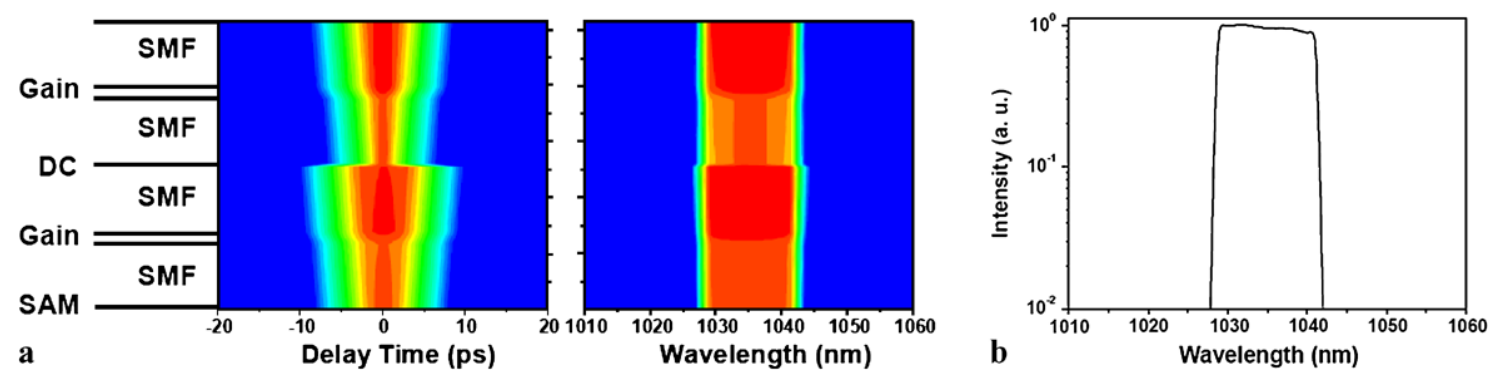

Fig. 11 (a) Simulation of the intra-cavity wave-breaking-free pulse evolution in the spectral and temporal domain. (SAM: saturable-absorber mirror, SMF: passive single mode fiber, DC: chirped FBG for dispersion compensation), (b) spectrum at the output port. (Logarithmic scale: $-30 \mathrm{~dB}=10 \mathrm{~dB}(\max ))$

Fig. 12 Optical spectrum of the stretched pulses in comparison with a parabolic, Gaussian and $\operatorname{sech}^{2}$ pulse shape
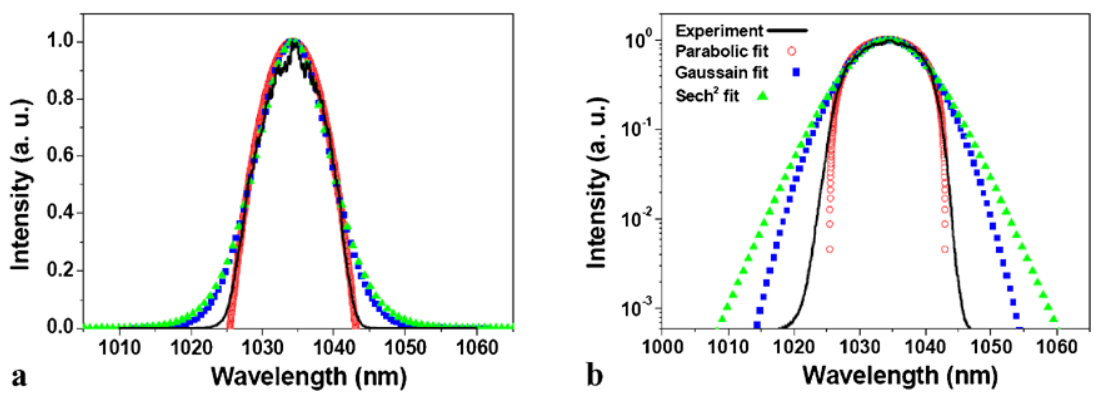

Fig. 13 (a) Autocorrelation trace of the chirped stretched-pulses.

(b) Autocorrelation trace of the compressed pulses using a HC-BGP fiber (solid line) compared to the transmission bulk grating compression (dashed line)

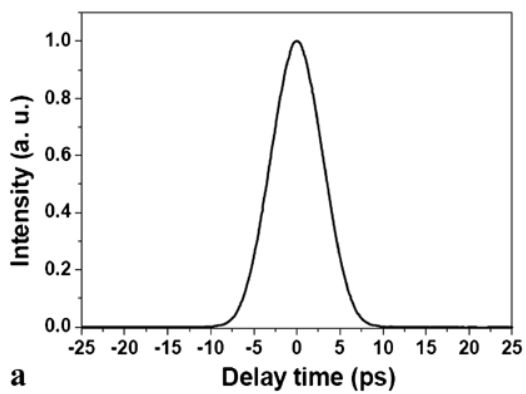

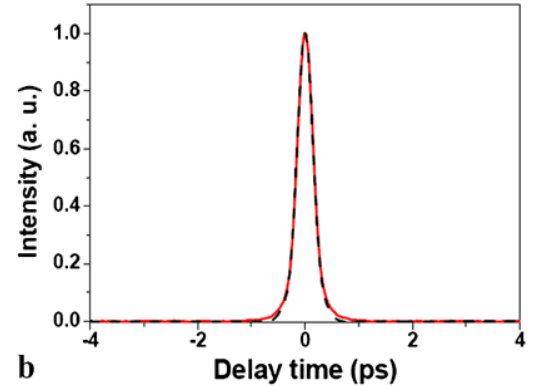

regime. First, to avoid of residual modulation in the spectrum, we used the PM-WDM outside the cavity and we pumped the doped fiber through the CFBG leading to a reduction of the intra-cavity splice issues [17]. The total fiber length inside the cavity is now $4.1 \mathrm{~m}$ resulting in a secondorder cavity dispersion of $0.016 \mathrm{ps}^{2}$. Therefore, the laser still operates in the normal-dispersion regime as in the above discussed configuration.

A self-starting and stable single-pulse train is generated and we could indeed produce a clean optical spectrum with very low residual modulation. Figure 12 shows the optical power spectrum of the output signal together with three different theoretical fits. The spectral profile of this regime fits well with a parabolic intensity profile near the peak over more than one decade of intensity. The pulses possess a spectral width of $12.3 \mathrm{~nm}$ and contain $160 \mathrm{pJ}$ of energy per pulse.

Figure 13(a) shows the autocorrelation trace directly obtained at the laser output. The positively chirped output pulses have a duration of 6.3 ps (autocorrelation width of $7.4 \mathrm{ps}$ ). The autocorrelation trace of the compressed pulses (Fig. 13(b)) using a HC-PBG fiber and the transmission bulk grating compression show the same autocorrelation width of $310 \mathrm{fs}$ (FWHM). It corresponds to a pulse duration of $213 \mathrm{fs}$, indicating a compression factor of more than 23 . The second-order dispersion, needed for pulse compression is $-0.17 \mathrm{ps}^{2}$. This value is very close but smaller than the negative dispersion given by the CFBG of $-0.19 \mathrm{ps}^{2}$. It therefore indicates that the pulses change the chirp sign from positive at the end of the normal GVD segment to negative at the end of the anomalous GVD segment. The autocorrelation of the transform-limited pulse calculated from the power spectrum as well as the measured autocorrelation traces are free from pedestal structures, what can be attributed to the weak residual modulation and the spectral shape in general (no steep edges). Consequently, due to the significantly cleaner compressed pulses this spectral shape of the pulses might be 

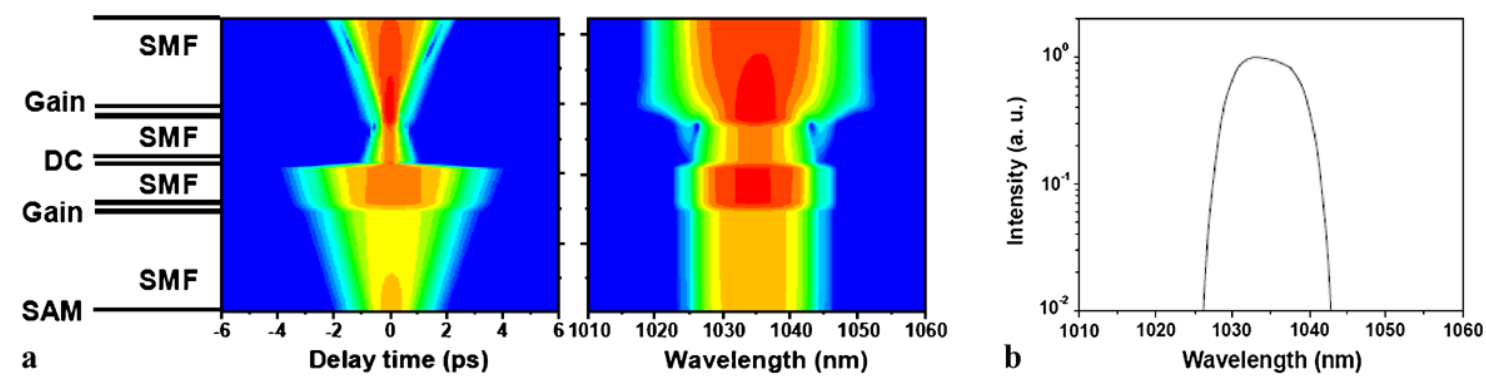

Fig. 14 (a) Simulation of the intra-cavity stretched-pulse evolution in the spectral and temporal domain. (SAM: saturable-absorber mirror, SMF: passive single mode fiber, $D C$ : chirped FBG for dispersion compensation), (b) spectrum at the output port. (Logarithmic scale: $-30 \mathrm{~dB}$ $0 \mathrm{~dB}(\max ))$

more attractive than the spectral shape obtained in the wavebreaking-free regime.

\subsubsection{Intracavity evolution}

The intra-cavity pulse evolution could be inferred from the magnitude of anomalous GVD required to dechirp the pulse outside the cavity. To study the pulse evolution the numerical simulation has been repeated with the parameters of the experimental setup in the second configuration. The result is shown in Fig. 14. This regime presents different temporal and spectral evolution of the pulses inside the cavity than in the previous regime. We can clearly see that the pulse spectrum changes dramatically during one round trip compared to the wave-breaking-free regime but also shows steep edges as observed experimentally. Two minima of the pulse duration are found located at the anomalous GVD segment and at the normal GVD segment, which indicates that the sign of the chirp reverses during one round trip. The minimum pulse duration is $371 \mathrm{fs}$. The pulse characteristics in the simulation show good agreement with the experiment. With these experimental and numerical results we can conclude that the laser works in a stretched-pulse regime with a parabolic spectral intensity profile, where the pulse has two minima inside the cavity with negative and positive chirp.

\section{New mode-locking regime at large positive dispersion with potential for stable high-energy operation-chirped-pulse fiber oscillator}

The dispersion issue in fiber-based mode-locked lasers plays an important role on pulse shaping and different pulse dynamics have been reported in various regimes of operation with a wide variety of pulse shapes. The fundamental soliton $\left(\mathrm{sech}^{2}\right)$ transform-limited pulses in the purely anomalous group velocity dispersion (GVD) fiber have been generated [18]. The dispersion-managed soliton regime operating in the anomalous net-cavity dispersion presents a similar spectral and temporal pulse shape with weak breathing inside the resonator [19]. As discussed above, approaching the zero net-cavity dispersion, the stretched-pulse regime is observed and Gaussian-shaped pulses experiences relatively large variations per cavity round trip [20]. In the normaldispersion regime, the monotonic frequency chirp evolution of the pulse is obtained by suppressing wave-breaking phenomena in the normal GVD cavity segment [16] and in a special case of this regime, the similariton laser, the output pulses are linearly chirped with a parabolic temporal intensity profile [5]. In addition to these cases, discussed so far, ultrashort pulse generation has also been realized in the purely normal-dispersion regime. Spectral filtering is applied to enhance self-amplitude modulation and a higher tolerance of accumulated nonlinear phase shifts has been obtained [21].

As a consequence of the above described approaches of dispersion management an increase of output pulse energy of fiber oscillators should be possible by incorporation of one or more segments possessing large positive GVD and no or negligible nonlinearity. This new approach will be investigated in the following sections.

\subsection{Pulse dynamics in the chirped-pulse fiber oscillator}

To investigate whether steady pulse solutions exist even at large additional positive group delay dispersion values, and to investigate the pulse evolution in this novel scheme, we set up the laser configuration shown in Fig. 15.

All fiber components are based on the polarizationmaintaining single-clad concept. A section of $30 \mathrm{~cm}$ highly ytterbium doped fiber is spliced between different lengths of passive fibers $(\mathrm{SMF} 1=1.2 \mathrm{~m}$ and SMF2 $=0.5 \mathrm{~m}$ ). One of the key elements in this cavity is the CFBG providing positive dispersion together with negligible nonlinearity. The dispersion and the peak reflectivity of CFBG have been measured to be $0.19 \mathrm{ps}^{2}$ (66\% of total-cavity dispersion) and $27 \%$ with Gaussian-like spectral bandwidth of $16 \mathrm{~nm}$, respectively. Several attractive properties of the CFBG can be employed as a highly positive dispersive element to stretch 


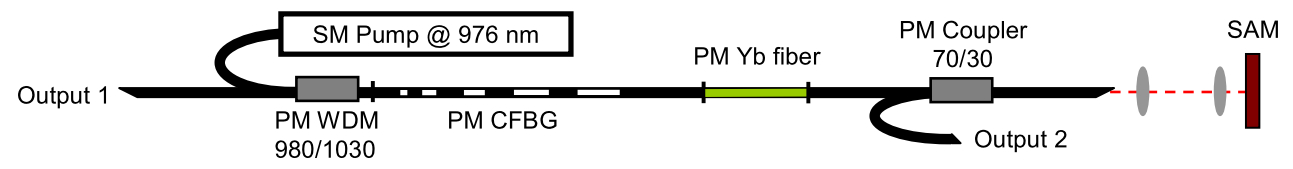

Fig. 15 Schematic representation of the passively mode-locked Yb-doped polarization-maintaining (PM) chirped-pulse fiber laser. SAM: saturable-absorber mirror, $C F B G$ : chirped fiber Bragg grating

Fig. 16 Results of experimentally measured optical spectrum (a) and autocorrelation trace of chirped pulses (b) from the fiber oscillator compared to numerical simulations (c-d). Solid line: output port 1, open circle: output port 2
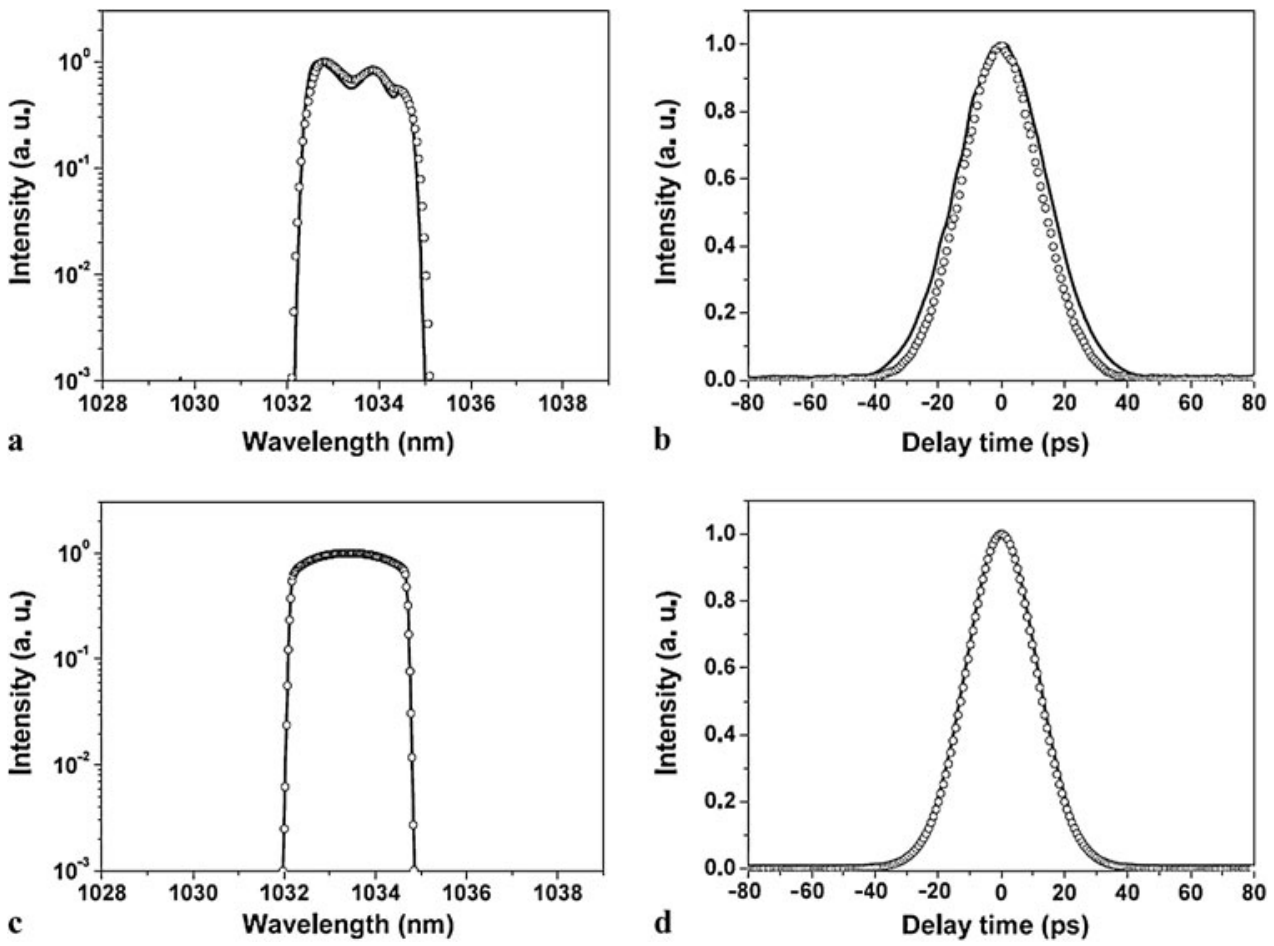

the pulse during its propagation and an output coupler (output 1) to study the laser operation. Passive mode-locking is achieved by using a semiconductor saturable absorber (for details see [22]). To study intra-cavity pulse dynamics and to select the single-polarization propagation in this laser configuration an additional fiber-based coupler (output 2) is inserted. The SAM and the fiber-based output coupler are placed at the end of the linear cavity. Self-starting and stable mode-locked operation is obtained by optimizing the saturation threshold on the SA for an adequate launched pump power. We investigate the intra-cavity pulse evolution in this configuration and the experimental results obtained at the two output ports are summarized in Fig. 16. The optical spectrum, shown in Fig. 16(a), obtained for a pump power of $155 \mathrm{~mW}$, possesses the same characteristics: steep spectral edges. The 10-dB and 3-dB bandwidths of the optical spectra at the output 1 (and output 2) are $2.4 \mathrm{~nm}(2.45 \mathrm{~nm}$ ) and $1.79 \mathrm{~nm}(2.05 \mathrm{~nm})$ respectively. By decreasing the pump power, the optical spectrum maintains the spectral profile but the spectral bandwidth decreases. The asymmetric spectral behavior can be attributed, at first, to the finite temporal response of the SA [23] and to the Bragg reflector behind the SA and the gain dynamic and transmission properties of the fiber based cavity elements (CFBG and coupler). An additional residual low frequency intensity modulation appears in the optical spectrum, which is very different than that reported in paragraph 2 where the presence of a high frequency intensity modulation is attributed to interference effects by polarization mode mixing and intra-cavity splice issues.

The autocorrelation traces obtained directly at the laser outputs are shown in Fig. 16(b). The positively chirped output pulses are well fitted with a Gaussian shape with pulse durations of $21.8 \mathrm{ps}$ and $19 \mathrm{ps}$. These pulses are externally compressed by a linear process outside the cavity (not shown in Fig. 15). The autocorrelation traces of the externally compressed pulse at both outputs present the same width of 2.1 ps (FWHM), which corresponds to a pulse duration of 1.37 ps. The transform-limited pulse duration is calculated to be $1.11 \mathrm{ps}$, which indicates that the pulses can be compressed down to near their transform-limited duration. The anomalous dispersion necessary for the compression of the chirped output pulses at the two output ports by the grating pair is more than $-7.7 \mathrm{ps}^{2}$. This indicates that the laser gen- 
erates highly positive chirped output pulses. We measured the average output powers of $33 \mathrm{~mW}$ and $3 \mathrm{~mW}$ at output 1 and 2, which corresponds to an energy per pulse of $750 \mathrm{pJ}$ and $68 \mathrm{pJ}$. The operation of mode-locking is very stable and self-starting with the same characteristics of temporal and spectral operation for equal pump power. Furthermore, the operation of the laser is characterized by very low amplitude noise.

We have experimentally presented the main properties of pulse evolution at the two different output ports. In order to obtain a better understanding of the pulse generation and the intra-cavity evolution of the highly chirped pulses in the above-reported laser configuration the laser has been studied numerically with the model from paragraph 1 . In the simulation the parameters for the cavity elements match those of the experimental setup. This includes the action of the output coupling, the saturable absorber, the active and passive fibers and an additional positive GVD segment with negligible nonlinearity and Gaussian-shaped spectral filter profile. The absorption in the semiconductor was described by the rate equation model [24]. The saturation energy due to the limited pumping is set in the model in such a way that the extracted energy is similar to that obtained in the experiments. The exact stable solution, obtained using quantum noise as the initial condition, is compared with the experimental results. Figure 16 also shows the spectral and temporal results of the numerical simulations obtained at the two output ports. One can clearly see that the pulse shows a steeply edged spectral profile as measured experimentally, as can be seen in Fig. 16(c) at both outputs. The spectral bandwidths at the output ports 1 and 2 are about $2.55 \mathrm{~nm}$ and $2.53 \mathrm{~nm}$. The simulated pulse durations at both output ports are present with the pulse widths of $18.5 \mathrm{ps}$ and $17.8 \mathrm{ps}$, and the pulse profiles are also best fitted with a Gaussian temporal intensity profile. However, in the chirped-pulse fiber laser presented here, the linear chirp is dominant in the numerical solution and recompression of the pulses close to the transform-limit is possible. The simulated pulse energy, duration, profile, spectral shape and bandwidth present very good agreement with the experimental results. It is therefore of great importance to understand better the physical mechanisms of pulse formation and evolution. To gain insight into the intra-cavity pulse evolution, the spectral and temporal characteristics of one cavity round trip of the pulse (stable solution after convergence) are shown in Fig. 17. As discussed above, we obtained positively chirped output pulses at both output ports. It should be mentioned that there is no negative GVD element implemented intra-cavity and the pulses are always positively chirped inside the cavity. The pulse duration increases monotonically within the gain fiber and passive fibers. We observe the local pulse stretching phenomenon in the CFBG segment. The total pulse shortening effect occurs in the SAM segment by the nonlinear absorbing mechanism. In the spectral domain, spectral

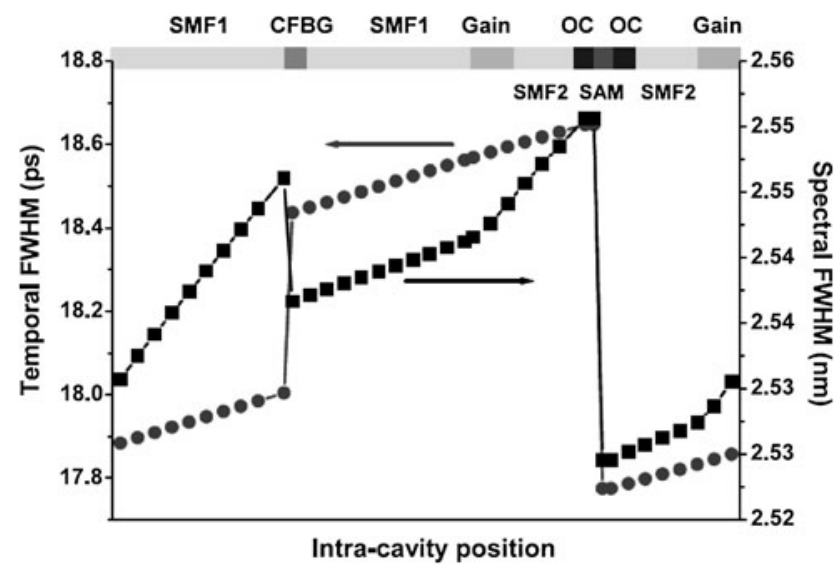

Fig. 17 Simulation of the intra-cavity pulse evolution of the mode-locked chirped-pulse fiber laser in the temporal and spectral domain. $O C$ : output coupler

broadening via self-amplitude modulation (SPM) can be observed during propagation through the fiber. An additional pulse shaping mechanism by spectral filtering is caused by the reflectivity properties of the CFBG, however, according to the simulation; this filtering is not needed for selfconsistency. The main spectral shortening effect occurred in the SAM segment. In addition, the simulation shows that spectral shaping by the gain profile is present even at that narrow spectral bandwidth. Finally, self-consistency could be achieved by the balance between the SAM nonlinearity, CFBG properties and the nonlinearity inside the gain and passive fibers. With these experimental and numerical results we can conclude that the pulse dynamics in the new regime reveals weak intra-cavity temporal and spectral breathing, leading to on average longer positively chirped pulses. Thus the output spectra and pulse profiles present similar shapes at the two opposite output ports.

Summarizing this section, we reported on a new nonlinear optical dissipative system in the form of a modelocked fiber laser operating in a highly positive dispersion regime. A segment possessing a large amount of positive GVD and negligible nonlinearity is added to an all-normal mode-locked fiber laser. A new pulse dynamic is demonstrated experimentally and numerically, which on average generates longer positively chirped pulses. The pulse evolution is characterized by weak intra-cavity temporal and spectral breathing with local temporal stretching phenomena. The interesting feature of the presented approach is that the limitations induced by the nonlinear effects could be reduced by scaling down the peak intensity inside the fiber core by stretching the pulse during the intra-cavity propagation leading to the possibility of energy scaling. 


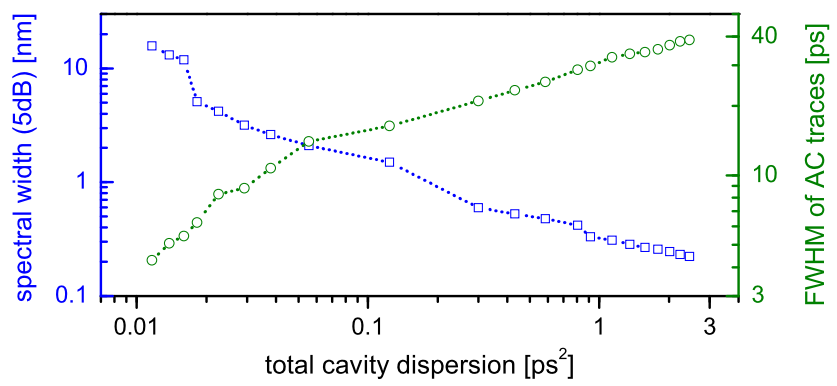

Fig. 18 Spectral width (blue, $\square$ ) and corresponding pulse durations $($ green,, ) over total cavity dispersion (TCD) for positive TCD values in logarithmic scale

4.2 Influence of total cavity dispersion in the chirped-pulse fiber oscillator

As mentioned above, one of the most important parameters influencing the system dynamics is the intra-cavity dispersion. Therefore following the above results an experimental study on the dispersion issue in the passively mode-locked chirped-pulse fiber oscillator concept was carried out. For this purpose a similar laser as presented in the previous section was set up. Instead of the CFBG a bulk transmission grating-based stretcher was implemented, which allowed varying the dispersion over a large range between $+2.4 \mathrm{ps}^{2}$ and $-1.6 \mathrm{ps}^{2}$ without adding additional nonlinearity.

Starting at one stable operation point, the total cavity dispersion (TCD) is varied while only the intra-cavity power is slightly adjusted to secure saturation of the SAM at longer pulse durations. Within the positive net-cavity dispersion operation chirped output pulses ranging from $4.3 \mathrm{ps}$ $@ 0.01 \mathrm{ps}^{2}$ TCD to 38.6 ps @2.4 ps $^{2}$ TCD are obtained. The spectral and temporal widths of the pulses, measured at the laser output are shown in Fig. 18. Using an external grating compressor the pulses could be compressed to durations between 292 fs @0.01 ps ${ }^{2}$ and 9 ps @ 1.2 ps $^{2}$, being close the respective transform limited durations. The used pump power remained at about $250 \mathrm{~mW}$, hence the width $(5 \mathrm{~dB})$ of the corresponding spectra decreased from $15.7 \mathrm{~nm}$ to $0.2 \mathrm{~nm}$ with increasing dispersion and increasing pulse durations, due to the fact that the influence of self phase modulation (SPM) is decreasing with decreasing peak power. This decrease in bandwidth leads to saturation of the effective pulse stretching for large GVD values (Fig. 18).

Similar behavior is observed in the negative dispersion region, where the spectral width $(5 \mathrm{~dB})$ decreases from $5.29 \mathrm{~nm} @-0.07 \mathrm{ps}^{2}$ to $0.64 \mathrm{~nm} @-1.57 \mathrm{ps}^{2}$, whereas the pulse durations increase from 0.8 ps to 3.9 ps (not shown in Fig. 18). Near zero-dispersion the oscillator operates in double pulse regimes [25] at high pump power. With longer pulse durations at large negative dispersion the peak power decreases and therefore nonlinear effects play a minor role where we obtained maximum output pulse energies (close to nanojoule) at maximum available pump power $(270 \mathrm{~mW})$.
With this configuration stable mode-locking in the extremely high anomalous dispersion $\left(-1.6 \mathrm{ps}^{2}\right)$ is demonstrated. Furthermore operation in the chirped-pulse regime at extremely high total cavity dispersion $\left(+2.4 \mathrm{ps}^{2}\right)$ is evinced. In both extreme cases very low breathing inside the cavity is observed. Due to solitary pulse formation at anomalous dispersion the pulse stretching is much lower than at high normal net-cavity dispersion. As consequence of the very long pulse durations at high positive dispersion the nonlinear phase accumulated during one round trip is reduced about an order of magnitude compared to near-zero net-cavity dispersion. Pulse shaping is governed by the nonlinear temporal action of the semiconductor saturable absorber, which compensates the local stretching, introduced by the dispersion management.

\subsection{Route to extreme pulse energies in a chirped-pulse fiber laser}

As many applications require high-peak power pulses, there is a strong interest in oscillators, generating ultrashort pulses with high energy. Excessive Kerr-nonlinearity hinders the self-consistent pulse evolution in a resonator round trip, which makes high-energy pulse generation particularly challenging in mode-locked fiber oscillators. As known from ultrafast fiber amplifier systems a reduction of nonlinearity and consequently potential performance enhancement can be obtained by the enlargement of the fiber mode area while maintaining single-mode guidance. The use of microstructured fibers enabled fiber oscillator designs with ultralow nonlinearity. The increased mode area decreases the intensity of the light field, additionally the interaction length can be significantly reduced by reducing the fiber section in the cavity to the extreme case of solely a short piece of gain fiber. Exploiting the advantages of microstructured fibers together with high-energy dissipative soliton pulse shaping a tremendous performance increase in terms of pulse energy, peak power and also average power has been obtained [26-29].

In this section, we report on the investigation of energy scaling in mode-locked fiber oscillators based on recent experimental results. Our numerical simulation is done with the model described in 1.1. The simulated oscillator scheme is shown in Fig. 19, the parameters for each element match those of the experimental setup in [29], where the generation of microjoule-class pulses is demonstrated in a low nonlinearity large mode-area (LMA) photonic crystal-fiber (PCF) oscillator without dispersion compensation. Calculated pulse solutions show very good agreement with the experimental data [29]. Guided by the above results, we applied the chirped pulse concept to the LMA-PCF laser by incorporation of a positive dispersive element with negligible nonlinearity directly in front of the gain fiber, in order to achieve further energy and peak power scaling. 
Fig. 19 Simulated chirped-pulse fiber oscillator cavity scheme

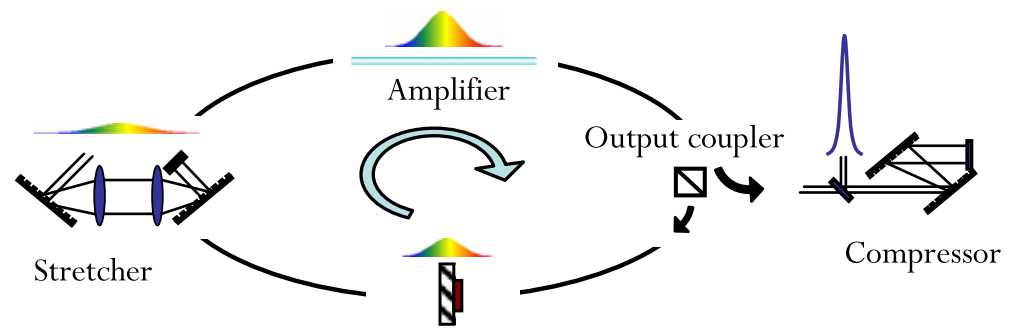

Semi-conductor saturable absorber
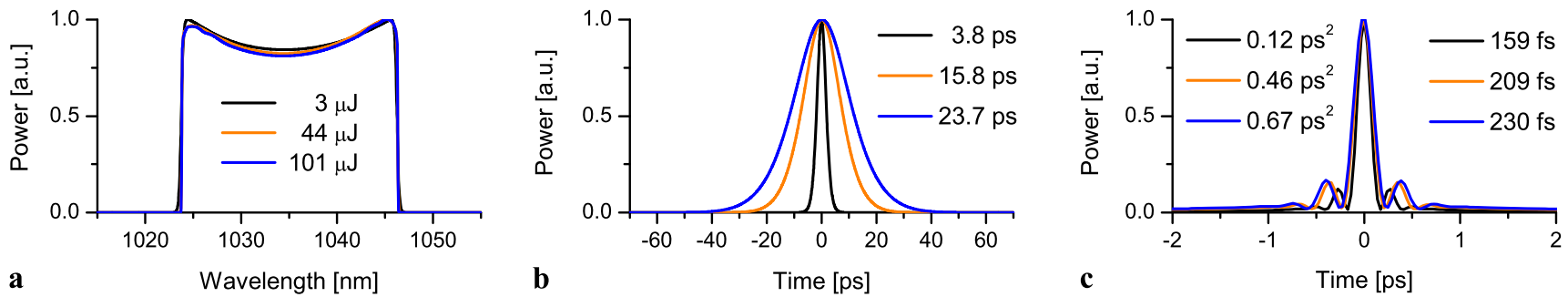

Fig. 20 Spectral (a) and temporal pulse shape of the chirped (b) and extra-cavity compressed output pulses (c)

Beginning with the self-starting solution at $\sim 1 \mu \mathrm{J}$, which reproduces the experimental results, we increased only the saturation energy until an output pulse energy of $3 \mu \mathrm{J}$ was obtained at the laser output. The spectrum, which increased in bandwidth (to $23 \mathrm{~nm}$ ), and the pulse shape at the output are shown in Fig. 20 (black). At this operation point, we apply the chirped-pulse concept by increasing the amount of additional dispersion and pump power (more specifically $\left.E_{\text {sat }}\right)$ simultaneously in such manner that the pulse bandwidth at the laser output remained unchanged $(23 \mathrm{~nm})$. This way, the output pulse energy could be increased to the $100 \mu \mathrm{J}$ level (Fig. 21). The pulse duration increased linearly up to 24 ps with increasing dispersion value (Fig. 22). This corresponds to stretching factors up to $\sim 150$ toward the transform limit. By removing only the linear chirp, peak powers of more than $200 \mathrm{MW}$ become accessible. Output pulse shapes change little with increasing dispersion and energy; only the spectral edges become more emphasized, which can be seen in Fig. 20, where the spectral and temporal shape at three representative energy levels are shown.

Due to the reduced spectral broadening effect by SPM on highly chirped pulses, the maximal peak power inside the cavity is found to increase linearly with dispersion (Fig. 21). Hence, limiting factors for the energy scaling of femtosecond pulses arise. The self-focusing limit at $4.3 \mathrm{MW}$ in fused silica will play a role at pulse energies above $100 \mu \mathrm{J}$. Furthermore increasing nonlinear phase shifts at high intra-cavity peak powers will destabilize the mode-locking. As a result of increasing nonlinear chirp an increase in compressed pulse duration is observed (Fig. 22) together with a decrease in pulse quality in high-energy operation (Fig. 20(c)). Please, note that these high energy strongly stretched pulse solutions are self-sustaining, but not self-starting from noise.

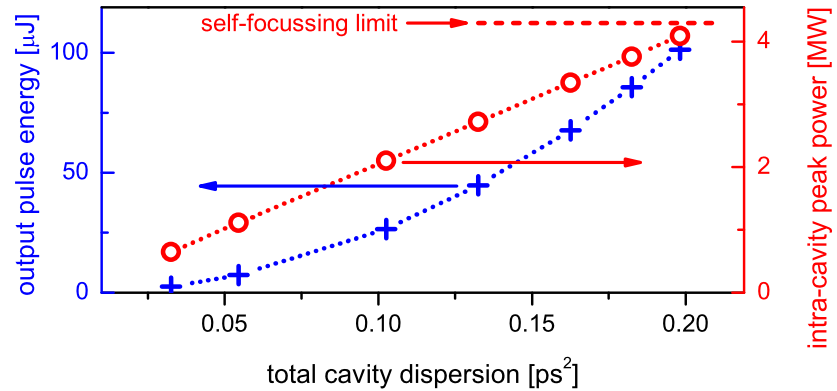

Fig. 21 Extracted energy (blue, + ) and maximum intra-cavity peak power $(r e d, \circ)$ over total cavity dispersion

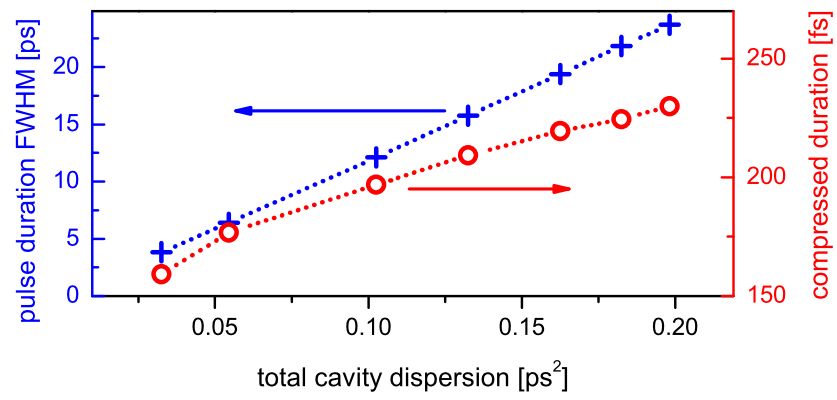

Fig. 22 Output pulse duration (blue, + ) and extra-cavity compressed pulse duration $(r e d, \circ)$ over total cavity dispersion

Thus experimental access would require the above described simultaneous increase of pump power with dispersion, starting from a stable mode-locked low energy state.

Inside the cavity the spectral breathing is low $(<2 \%$ changes in spectral FWHM), whereas the temporal breathing increases for higher pulse energies, as gain dispersion [30] plays a key role in this region. Due to the broad spec- 


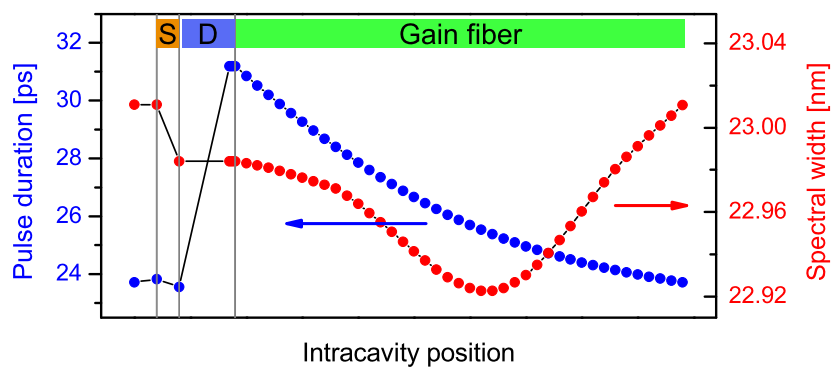

Fig. 23 Intra-cavity pulse evolution for the $101 \mu \mathrm{J}$ case, $S$ : semiconductor saturable absorber, $D$ : dispersive stretcher

trum and strong amplification the pulse evolution is different from the cases discussed before, the influence of the saturable absorber is decreased and temporal shaping is governed by the local linear stretching of the dispersive element and the shaping due to the limited gain bandwidth inside the fiber. The evolution of the temporal and spectral pulse width in high-energy operation is summarized in Fig. 23.

\section{Conclusion}

In conclusion, we have presented an experimental and numerical study on the nonlinear pulse dynamics in passively mode-locked fiber laser systems. We have shown that for the non-distributed model to describe a mode-locked fiber laser, multiple attractors are accessible by different initial conditions even for fixed system parameters. Different regimes of mode-locking obtained by varying the intra-cavity dispersion and saturation energy of the gain fiber have been carefully analyzed. It is shown that self-similar evolution leading to linearly chirped parabolic pulses occurs for a parameter range that is embedded in a more general regime of wave-breaking-free operation. Stretched-pulse and wavebreaking-free operation have been experimentally demonstrated in an environmentally stable $\mathrm{Yb}$-doped all-fiber laser. The completely alignment-free, ultracompact and robust femtosecond laser source has great potential for several applications. In particular, clean chirped pulses with parabolic spectral profile are generated in the stretched-pulse regime. The pulse evolution in the different operation modes is studied with the non-distributed numerical model. Furthermore a new operation mode at high positive cavity dispersion is reported. Detailed experimental and numerical studies of the main properties of the pulse shaping mechanism are presented. A highly positive GVD, negligible nonlinearity segment is implemented in the all-normal fiber laser concept and the local temporal stretching phenomenon is observed during the intra-cavity propagation. Mode-locked operation over a large dispersion range is investigated in an experimental chirped-pulse oscillator scheme. Highly positively chirped pulses with very weak intra-cavity temporal and spectral changes, and consequently, on average longer pulses and lower peak powers have been generated. The interesting feature of the presented approach is that the limitations induced by the nonlinear effects could be reduced by scaling down the peak intensity inside the fiber core by stretching the pulse during the intra-cavity propagation leading to the possibility of energy scaling. The performance capability of this approach in combination with a large-modearea fiber is numerically studied and reveals enormous scaling potential.

Acknowledgement This work was supported by the Deutsche Forschungsgemeinschaft (Research Group "Nonlinear spatio-temporal dynamics in dissipative and discrete optical systems", FG 532).

\section{References}

1. N. Akhmediev, A. Ankiewicz, Dissipative Solitons. Lecture Notes in Physics, vol. 661 (Springer, Berlin, 2005)

2. H.A. Haus, IEEE J. Sel. Top. Quantum Electron. 6, 1173 (2000)

3. I.P. Christov, M.M. Murnane, H.C. Kapteyn, J. Zhou, C.-P. Huang, Opt. Lett. 19, 1465 (1994)

4. H.A. Haus, K. Tamura, L.E. Nelson, E.P. Ippen, IEEE J. Quantum Electron. 31, 591 (1995)

5. F.Ö. Ilday, J. Buckley, W. Clark, F. Wise, Phys. Rev. Lett. 92, $213902(2004)$

6. T. Schreiber, B. Ortaç, J. Limpert, A. Tünnermann, Opt. Express 15, 8252 (2007)

7. A. Ruehl, O. Prochnow, D. Wandt, D. Kracht, B. Burgoyne, N. Godbout, S. Lacroix, Opt. Lett. 31, 2734 (2006)

8. M.E. Ferman, V.I. Kruglov, B.C. Thomsen, J.M. Dudley, J.D. Harvey, Phys. Rev. Lett. 84, 6010 (2000)

9. V.I. Kruglov, A.C. Peacock, J.D. Harvey, J.M. Dudley, J. Opt. Soc. Am. B 19, 461 (2002)

10. T. Schreiber, C.K. Nielsen, B. Ortac, J. Limpert, A. Tünnermann, Opt. Lett. 31, 574 (2006)

11. C. Nielsen, B. Ortaç, T. Schreiber, J. Limpert, R. Hohmuth, W. Richter, A. Tünnermann, Opt. Express 13, 9346 (2005)

12. L. Lefort, J. Price, D. Richardson, G. Spühler, R. Paschotta, U. Keller, A. Fry, J. Weston, Opt. Lett. 27, 291 (2002)

13. B. Ortaç, A. Hideur, T. Chartier, M. Brunel, C. Özkul, F. Sanchez, Opt. Lett. 28, 1305 (2003)

14. A. Albert, V. Couderc, L. Lefort, A. Barthélémy, IEEE Photonics Technol. Lett. 16, 416 (2004)

15. F.Ö. Ilday, J. Buckley, H. Lim, F.W. Wise, W. Clark, Opt. Lett. 28, $1365(2003)$

16. B. Ortaç, M. Plötner, T. Schreiber, J. Limpert, A. Tünnermann, Opt. Express 15, 15595 (2007)

17. I. Hartl, G. Imeshev, L. Dong, G.C. Cho, M.E. Fermann, in Conference on Lasers and Electro-Optics/Quantum Electronics and Laser Science and Photonic Applications Systems Technologies, Technical Digest $(C D)$ (Optical Society of America, Washington, 2005), paper CThG1

18. I.N. Duling III, Opt. Lett. 16, 539 (1991)

19. B. Ortaç, J. Limpert, A. Tünnermann, Opt. Lett. 32, 2149 (2007)

20. K. Tamura, E.P. Ippen, H.A. Haus, Appl. Phys. Lett. 67, 158 (1995)

21. A. Chong, W.H. Renninger, F.W. Wise, Opt. Lett. 32, 2408 (2007)

22. B. Ortaç, M. Plötner, J. Limpert, A. Tünnermann, Opt. Express 15, 16794 (2007)

23. A. Cabasse, B. Ortaç, G. Martel, A. Hideur, J. Limpert, Opt. Express 16, 19322 (2008) 
24. N.N. Akhmediev, A. Ankiewicz, M.J. Lederer, B. Luther-Davies, Opt. Lett. 23, 280 (1998)

25. G. Martel, C. Chédot, V. Reglier, A. Hideur, B. Ortaç, P. Grelu, Opt. Lett. 32, 343 (2007)

26. M. Baumgartl, B. Ortaç, C. Lecaplain, A. Hideur, J. Limpert, A. Tünnermann, Opt. Lett. 35, 2311 (2010)

27. C. Lecaplain, B. Ortaç, G. Machinet, J. Boullet, M. Baumgartl, T. Schreiber, E. Cormier, A. Hideur, Opt. Lett. 35, 3156 (2010)
28. M. Baumgartl, F. Jansen, F. Stutzki, C. Jauregui, B. Ortaç, J. Limpert, A. Tünnermann, Opt. Lett. 36, 244 (2011)

29. B. Ortaç, M. Baumgartl, J. Limpert, A. Tünnermann, Opt. Lett. 34, 1585 (2009)

30. L.M. Zhao, C. Lu, H.Y. Tam, P.K.A. Wai, D.Y. Tang, Appl. Opt. 48, 5131 (2009) 